\title{
DESERCIÓN ESTUDIANTIL EN LA FACULTAD DE ENFERMERÍA DE LA FUNDACIÓN UNIVERSITARIA DE CIENCIAS DE LA SALUD
}

Luisa Fernanda Cortés V., Marianella Cruz H., Gloria Maritza Pinilla P.*

\section{Resumen}

El trabajo se realizó con el animo de indagar a cerca de los factores que intervienen en la deserción estudiantil, en la Facultad de Enfermería de la Fundación Universitaria de Ciencias de la Salud (FUCS) y buscar posibles alternativas de solución que puedan brindarse a la institución con el fin de cautivar a los estudiantes dentro de ella. Se aplicó un instrumento estructurado a 20 desertores que habían dejado la facultad por razones diferentes al nivel académico (exestudiantes con promedios académicos inferiores a los preestablecidos por la institución y aquellos que no tuvieron la oportunidad de nivelar asignaturas, ya que no se contemplaba dentro del reglamento), que son reconocidos factores de deserción estudiantil, por lo que se considera esencial ahondar en este tema.

\section{Introduccíón}

Con este trabajo se buscó demostrar que el abandono de los estudiantes no esta dado por voluntad simplista del estudiante, como tampoco por la rigurosidad disciplinaria del reglamento académico existente. A cada institución universitaria se le reconoce como un espacio de reflexión sobre los problemas del conocimiento y de sus aplicaciones, pero a veces se desconoce su propia realidad, lo cual lleva a rupturas culturales y académicas que, con frecuencia, se asumen con formas agresivas a largo o mediano plazo deteriorando la calidad de vida de los estudiantes, quienes se van desmotivando con expresiones de cansancio, de no hallarse consigo mismo, de no comprender el mundo que les ofrece la universidad. La deserción estudiantil se atribuye a factores multicausales siendo más importantes los de orden emocional y comunicativo (estrés, relación docente-alumno, académico y socioeconómico).

Facultad de Enfermería. Fundación Universitaria de Ciencias de la Salud, Hospital de San José, Bogotá, D.C., Colombia
Desertar simboliza y conlleva la idea de error y la consecuente noción de sanción, castigo, reprimenda institucionalizada, social y familiar. Dentro de la deserción se diferencian dos tipos: la voluntaria, como resultado de una decisión propia del estudiante para dejar la carrera por factores endógenos o exógenos al mismo sistema y la obligatoria, generada por factores psicosociales o académicos que fomentan el abandono por parte de los estudiantes.

La educación superior es el proceso por el cual cada uno de los individuos se apropia del saber colectivo que han desarrollado generaciones anteriores en todas las latitudes y las utiliza para sí mismo y para sus tareas. Las universidades tienen el reto de aportar a la formación de una sociedad con capacidades para el aprovechamiento científico y tecnológico, requisitos para una presencia competitiva en el ámbito internacional y aportar a la construcción de una sociedad más justa y productiva. El compromiso de las instituciones de educación superior con la formación de recursos humanos y el desarrollo deben conducir a la definición de políticas y progra- 
mas que coadyuven a la generación de ciencia y tecnología y a la formación axiológica de los ciudadanos que la modernidad reclama.

Ahora más que nunca, la calidad de la educación no está asociada a la acumulación de información. La formación integral del ser humano busca su crecimiento como individuo capaz de asimilar, transformar, crear y asumir una postura crítica y autónoma frente a sí mismo, con objetivos prioritarios por lograr. Una educación es de calidad en la medida en que satisfaga las necesidades sociales de la comunidad humana específica a la cual se da y a la cual se sirve. Por lo tanto, la alta calidad en la educación es determinante en la preparación de ciudadanos responsables y en la formación académica integral.

\section{Razones de deserción estudiantil}

En este tema se estudiaron una serie de elementos que por desconocimiento se creían inexistentes, quizás con el ánimo inconsciente de reducir su complejidad, más aún si la mayoría de las causas se dirigen al abandono de los estudiantes de la Fundación Universitaria de Ciencias de la Salud por motivos no académicos.

Algunos lo hacen por razones personales, generadas por su entorno social y que influyen parcial o totalmente en la decisión de abandonar la universidad, otros por razones familiares; al respecto, es importante prever que la familia directa e indirectamente tienen gran influencia en la decisión de abandonar la universidad, por lo que busca aportar elementos sobre características y dificultades que el estudiante identifica en su desempeño intelectual en actividades de carácter académico cotidiano.

En cuanto a las razones académicas, se analizan características personales e institucionales que de una u otra forma están estrechamente ligadas y que afectan la permanencia del estudiante en la entidad educativa (aptitud, orientación, elección de la carrera o institución, rendimiento académico, formación previa), y dentro de los socioeconómicos existe una relación, aunque no siempre directa y evidente, entre las características sociales, económicas y culturales de los estudiantes con la relación que estos establecen con la universidad, expresadas en las diferentes formas de enfrentar el trabajo y lo que ello implica. Es de resaltar que existe una línea general equivocada al buscar la falla en el estudiante, quien comete errores por características endógenas que lo llevan a chocar muy fácilmente con el sistema, desajustado por elementos que le son propios.

\section{Metodología}

El problema de investigación se trabajó con una metodología cuantitativa. La población seleccionada fueron los estudiantes que habían dejado la facultad de Enfermería de la FUCS, entre el primer periodo de 1996 y el segundo periodo de 1999. El tipo de investigación fue descriptivo, ya que se mostraron los resultados tal como se presentaron y no solamente se dieron a conocer estadísticas acerca de los factores sino que se buscaron posibles soluciones para disminuir el índice de deserción en la institución.

\section{Análisis de los resultados}

1. Al ingresar a la universidad, 16 personas se encontraban entre las edades de 18 a 25 años, lo que hace pensar que contaban con una madurez intelectual adecuada, pero tenían un atraso aproximado de cuatro años por edad para ingresar a la educación superior, según lo reafirma el autor Tinto Vincent.

2. Durante la permanencia de los estudiantes en la universidad, 18 refirieron que sus padres estaban con vida, lo que se refleja en una estabilidad emocional, que influye sustancialmente en la permanencia en la institución, según Tinto Vincent.

3. El tiempo entre la culminación del bachillerato y el ingreso a la universidad fue de uno a dos años para 10 persona, lo que influye en la decisión en el momento de elegir la carrera.

4. Para 16 personas, las actividades que realizaron durante este tiempo fueron diferentes a las op- 
ciones dadas en el cuestionario, lo que se refleja al momento de elegir la carrera.

5. Seis personas ingresaron a la FUCS por voluntad propia, lo que muestra que estaban bien encaminadas en la carrera elegida.

6. Ingresaron a la FUCS porque el perfil era diferente al de otras universidades; esto indica que conocían la trayectoria de la FUCS.

7. Para 16 personas, la FUCS no les ofreció la oportunidad de explorar otras áreas diferentes a las de la carrera, lo que influye en la permanencia de los estudiantes dentro de la institución ya que se ven absorbidos sólo por áreas de la carrera.

8. Para 11 personas, esta era la carrera que debían estudiar, lo cual demuestra seguridad en el momento de elegir la carrera (Tabla 1).

\section{Información familliar}

1. Al ingresar a la FUCS, ocho personas tenían por 1o menos dos hermanos de los cuales, al menos uno se encontraba estudiando, lo que influye en la estabilidad económica del hogar ayudado esto por la crisis que cursa el país.

2. Para ocho personas, el lugar que ocupaban entre sus hermanos era el segundo, lo que se refleja en la estabilidad en el hogar, ya que esto demanda más gastos en los hogares.

3. De los encuestados, 18 sujetos manifestaron tener apoyo frente a la carrera, lo que influye en la permanencia del estudiante al no ir en contra de ideales y creencias que se tienen en el hogar.

4. Al ingresar a la universidad, 18 de los encuestados eran solteros lo que indica que no tenían compromisos que intervinieran en sus anhelos al ser jefes de hogar (Tabla 2).

\section{Información institucional}

1. Para 10 de los encuestados, el sitio de ubicación de la FUCS era alejado de sus viviendas y esto influye en la permanencia por mayor tiempo de desplazamiento hacia la institución.

2. Para 19 personas, la universidad no le brindó campos adecuados para la recreación, lo que se ve reflejado en el inconformismo de los estudiantes y se expresa con aburrimiento por su enfoque en la parte académica.

3. Diez de las personas encuestadas pensaban que el reglamento de la FUCS era riguroso, lo que indica que no existía una integración que estimulara la conciencia individual y colectiva (Tabla 3).

\section{Información académica}

1. De los encuestados, 16 realizaron el bachillerato en la jornada diurna; esto indica que hay una adecuada adaptación a la jornada educativa que se maneja en la FUCS.

2. Quince personas escogieron enfermería por inclinación personal, lo cual refleja seguridad y autonomía en la toma de decisiones.

3. La percepción que tenían del nivel académico de la universidad y los docentes era alto para 18 personas; esto hace pensar que la institución no es la que esta errando en el área de capacitación de sus educandos y de sus educadores.

4. Para 18 personas la universidad no les ofreció la oportunidad de alguna orientación en los momentos de inseguridad y problemas a los que se enfrenta el estudiante durante su permanencia en la institución (Tabla 4).

\section{Información socioeconómica}

1. Al ingreso, 13 personas dependían totalmente de sus padres, lo que hace pensar que en el momento en que existiera una inestabilidad en el hogar, ellos eran los directamente perjudicados.

2. Al ingresar a la universidad, 6 personas estudiaban $y$ trabajaban, y ello se refleja en carga laboral y académica y afecta su permanencia en la institución. 


\section{Tabla 1}

INFORMACIÓN PERSONAL

\begin{tabular}{|c|c|c|c|c|c|}
\hline ASPECTOS & \multicolumn{4}{|c|}{ OPCIONES DE RESPUESTA } & TOTAL \\
\hline Edad al ingresar & $<$ de 18 años & Entre $18-25$ & $>26$ años & & \multirow[b]{2}{*}{20} \\
\hline a la universidad & 3 & 16 & 1 & & \\
\hline \multirow{2}{*}{$\begin{array}{l}\text { Durante la permanencia } \\
\text { en la U. sus padres vivían }\end{array}$} & Los dos & La madre & El padre & Ninguno & \multirow[b]{2}{*}{20} \\
\hline & 18 & 2 & 0 & 0 & \\
\hline \multirow{2}{*}{$\begin{array}{l}\text { Cuál fue el tiempo que } \\
\text { transcurrió entre el ingreso } \\
\text { a la U.y la culminación } \\
\text { del bachillerato }\end{array}$} & Inmediato & Entre $1-2$ años & De 2-3 años & Más de 3 años & \multirow[b]{2}{*}{20} \\
\hline & 8 & 10 & 2 & 0 & \\
\hline \multirow{2}{*}{$\begin{array}{l}\text { Qué actividades realizó } \\
\text { durante este tiempo }\end{array}$} & Recreativas & Culturales & Políticas & Otra & \multirow[b]{2}{*}{20} \\
\hline & 3 & I & 0 & 16 & \\
\hline \multirow{2}{*}{$\begin{array}{l}\text { Cuándo ingresó a } \\
\text { la U. lo hizo por }\end{array}$} & Voluntad Propia & Influencia familiar & Estatus social & Otro & \multirow[b]{2}{*}{20} \\
\hline & 16 & 3 & 1 & 0 & \\
\hline \multirow{2}{*}{$\begin{array}{l}\text { Cuándo ingresó a la U. } \\
\text { le fue brindada alguna } \\
\text { información acerca de }\end{array}$} & Duración carrera & Plan curricular & $\begin{array}{c}\text { Proyecciones } \\
\text { futuras }\end{array}$ & Otro & \multirow[b]{2}{*}{20} \\
\hline & 8 & 7 & I & 7 & \\
\hline \multirow{2}{*}{$\begin{array}{l}\text { Ingresó a la FUCS por } \\
\text { que el perfil era diferente } \\
\text { a otras Universidades }\end{array}$} & Sí & No & Otro & & \multirow[b]{2}{*}{20} \\
\hline & 19 & 1 & 0 & & \\
\hline \multirow[b]{2}{*}{$\begin{array}{l}\text { La U. le ofreció la } \\
\text { oportunidad de } \\
\text { explorar otra área } \\
\text { diferente a las de la } \\
\text { carrera en tiempo } \\
\text { extracurricular }\end{array}$} & Sí & No & Otro & NS/NR & \multirow[b]{2}{*}{20} \\
\hline & 3 & 16 & 0 & 1 & \\
\hline \multirow{2}{*}{$\begin{array}{l}\text { Usted trabajaba } \\
\text { contemplaba la posibilidad de }\end{array}$} & Mejorar estatus & Superación personal & Otro & NS/NR & \multirow[b]{2}{*}{20} \\
\hline & 3 & 2 & 9 & 3 & \\
\hline \multirow{2}{*}{$\begin{array}{l}\text { El retiro de la universidad } \\
\text { le permitió ver que esa no } \\
\text { era la carrera que debía } \\
\text { estudiar }\end{array}$} & Sí & No & Otro & & \multirow[b]{2}{*}{20} \\
\hline & 8 & II & 1 & & \\
\hline
\end{tabular}




\section{Tabla 2}

\section{INFORMACIÓN FAMILIAR}

\begin{tabular}{|c|c|c|c|c|c|}
\hline \multirow{3}{*}{$\begin{array}{l}\text { ASPECTOS } \\
\text { La formalidad conyugal } \\
\text { de sus padres era: }\end{array}$} & \multicolumn{4}{|c|}{ OPCIONES DE RESPUESTA } & \multirow{3}{*}{$\begin{array}{l}\text { TOTAL } \\
20 \\
\end{array}$} \\
\hline & Casados & Viudos & Unión libre & Separado & \\
\hline & 15 & 1 & 4 & 0 & \\
\hline \multirow{2}{*}{$\begin{array}{l}\text { Cuántos hermanos tenía } \\
\text { al ingresar } \\
\text { a la universidad }\end{array}$} & Uno & Dos & Más de dos & Ninguno & \multirow[b]{2}{*}{20} \\
\hline & 5 & 8 & 6 & 1 & \\
\hline \multirow{2}{*}{$\begin{array}{l}\text { Qué lugar ocupó entre } \\
\text { sus hermanos }\end{array}$} & Hijo único & Primero & Segundo & Medio & \multirow[b]{2}{*}{20} \\
\hline & 0 & 7 & 8 & 5 & \\
\hline \multirow{2}{*}{$\begin{array}{l}\text { Al ingresar a la universidad } \\
\text { cuántos de sus hermanos } \\
\text { estudiaban }\end{array}$} & Uno & Dos & Más de dos & Ninguno & \multirow[b]{2}{*}{20} \\
\hline & 8 & 3 & 7 & 2 & \\
\hline \multirow{2}{*}{$\begin{array}{l}\text { Al ingresar a la universidad } \\
\text { recibía apoyo de su familia } \\
\text { frente a la carrera }\end{array}$} & Sí & No & Otro & & \multirow[b]{2}{*}{20} \\
\hline & 18 & 1 & I & & \\
\hline \multirow{2}{*}{$\begin{array}{l}\text { Al ingresar a la universidad } \\
\text { cuál era su estado civil }\end{array}$} & Soltero & Casado & Unión libre & Separado & \multirow[b]{2}{*}{20} \\
\hline & 19 & 0 & 1 & 0 & \\
\hline
\end{tabular}

\section{Tabla 3}

INFORMACIÓN INSTITUCIONAL

\begin{tabular}{l|c|c|c|c|c}
\hline \multirow{2}{*}{ ASPECTOS } & \multicolumn{3}{|c|}{ OPCIONES DE RESPUESTA } & \multirow{2}{*}{ TOTAL } \\
\hline \multirow{2}{*}{$\begin{array}{l}\text { El lugar de ubicación de } \\
\text { la universidad era }\end{array}$} & Fácil acceso & Alejado de la vivienda & Inseguro & Otro & \multirow{2}{*}{20} \\
\cline { 2 - 5 } & 3 & 10 & 6 & 0 & \multirow{2}{*}{20} \\
\cline { 1 - 5 } $\begin{array}{l}\text { La U. Le brindó campos } \\
\text { adecuados para } \\
\text { la recreación }\end{array}$ & Sí & No tro & 0 & \\
\hline $\begin{array}{l}\text { Durante el tiempo de } \\
\text { permanencia en la } \\
\text { universidad percibió } \\
\text { que el reglamento era: }\end{array}$ & Flexible & 19 & Inquebrantable & Otro & \multirow{2}{*}{20} \\
\cline { 2 - 5 } & 5 & 10 & 5 & 0 & \\
\end{tabular}




\section{Tabla 4}

\section{INFORMACIÓNACADEMICA}

\begin{tabular}{|c|c|c|c|c|c|c|}
\hline \multirow{3}{*}{$\begin{array}{l}\text { ASPECTOS } \\
\text { La jornada en que realizó } \\
\text { el bachillerato fue: }\end{array}$} & \multicolumn{5}{|c|}{ OPCIONES DE RESPUESTA } & \multirow{3}{*}{$\begin{array}{c}\text { TOTAL } \\
20 \\
\end{array}$} \\
\hline & Diurna & Nocturno & Valido & Semestralizado & Otro & \\
\hline & 16 & 3 & 0 & 0 & 0 & \\
\hline \multirow{2}{*}{$\begin{array}{l}\text { Qué lo llevo a escoger } \\
\text { enfermería como } \\
\text { posible carrera }\end{array}$} & $\begin{array}{l}\text { Influencia } \\
\text { Profesores }\end{array}$ & $\begin{array}{l}\text { Influencia } \\
\text { Amigos }\end{array}$ & $\begin{array}{c}\text { Inclinación } \\
\text { personal }\end{array}$ & $\begin{array}{l}\text { Presión } \\
\text { familiar }\end{array}$ & $\begin{array}{l}\text { Presión } \\
\text { empresa }\end{array}$ & \multirow[b]{2}{*}{20} \\
\hline & 0 & 0 & 15 & 5 & 0 & \\
\hline \multirow{2}{*}{$\begin{array}{l}\text { Qué percepción tenia } \\
\text { del nivel académico } \\
\text { de la universidad }\end{array}$} & Alto & Medio & Bajo & Otro & & \multirow[b]{2}{*}{20} \\
\hline & 18 & 2 & 0 & 0 & & \\
\hline \multirow{2}{*}{$\begin{array}{l}\text { Qué percepción tenía } \\
\text { del nivel académico } \\
\text { de los profesores }\end{array}$} & Alto & Medio & Bajo & Otro & & \multirow[b]{2}{*}{20} \\
\hline & 18 & 2 & 0 & 0 & & \\
\hline \multirow{2}{*}{$\begin{array}{l}\text { Durante la permanencia } \\
\text { en la universidad en } \\
\text { nivel académico se: }\end{array}$} & Mejoró & Mantuvo & Deterioró & NS/NR & & \multirow[b]{2}{*}{20} \\
\hline & 3 & 9 & 7 & 1 & & \\
\hline \multirow{2}{*}{$\begin{array}{l}\text { Antes de abandonar la } \\
\text { U. le brindaron alguna } \\
\text { información }\end{array}$} & Mejoró & Mantuvo & Deterioró & NS/NR & & \multirow[b]{2}{*}{20} \\
\hline & 3 & 9 & 7 & 1 & & \\
\hline \multirow{2}{*}{$\begin{array}{l}\text { Antes de abandonar la } \\
\text { U. le brindaron alguna } \\
\text { información }\end{array}$} & Sí & No & Otro & & & \multirow[b]{2}{*}{20} \\
\hline & 2 & 18 & 0 & & & \\
\hline
\end{tabular}

3. Cinco de las personas que laboraban se desempeñaban en áreas relacionadas con la carrera, de manera que estaban en pleno conocimiento del amor y dedicación que la enfermería implica.

4. Para ocho personas, las dificultades económicas no determinaron su retiro, ya que contaban con pleno apoyo de la familia y por consiguiente no se vieron afectadas por esto.

5. Aunque 15 personas no recibieron financiamiento de la carrera por parte de la institución, esto no fue un factor determinante en su retiro (Tabla 5).

\section{Conclusiones}

Durante la investigación no existió un factor prioritario en el retiro de quienes desertaron de la carrera y, por el contrario, se encontraron factores multicausales de la deserción estudiantil.

Contar con áreas que permitan la libertad de expresión de los alumnos es importante para no generar en ellos desmotivación, la cual se traduce en expresiones de aburrimiento y fatiga; de esta manera es posible enriquecer no sólo a los estudiantes sino a la facultad de enfermería. 


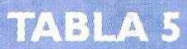

INFORMACIÓN SOCIOECONÓMICA

\begin{tabular}{|c|c|c|c|c|c|}
\hline ASPECTOS & \multicolumn{4}{|c|}{ OPCIONES DE RESPUESTA } & TOTAL \\
\hline Al ingresar a la U. recibía & Total & Parcial & No dependía & & \multirow[b]{2}{*}{20} \\
\hline $\begin{array}{l}\text { colaboración económica } \\
\text { de sus Padres }\end{array}$ & 13 & 5 & 2 & & \\
\hline \multirow{2}{*}{$\begin{array}{l}\text { Usted tuvo la necesidad } \\
\text { de trabajar }\end{array}$} & Antes de 16 años & Entre $17-25$ & No tuvo necesidad & & \multirow[b]{2}{*}{20} \\
\hline & 1 & 7 & 12 & & \\
\hline \multirow{2}{*}{$\begin{array}{l}\text { Si tuvo la necesidad } \\
\text { de trabajar fue por }\end{array}$} & Colaboración & Independencia & Otro & NS/NR & \multirow[b]{2}{*}{20} \\
\hline & 4 & 5 & 2 & 9 & \\
\hline \multirow[t]{2}{*}{ Al ingresar a la $U$. usted } & Estudiaba & Trabajaba & Est.udiaba y trabajaba & Ninguna & \multirow[b]{2}{*}{20} \\
\hline & 6 & 3 & 6 & 5 & \\
\hline \multirow{2}{*}{$\begin{array}{l}\text { Si se encontraba } \\
\text { trabajando se } \\
\text { desempeñaba en: }\end{array}$} & $\begin{array}{l}\text { Referente } \\
\text { a la carrera }\end{array}$ & $\begin{array}{l}\text { Relacionado } \\
\text { con la carrera }\end{array}$ & $\begin{array}{l}\text { Opuesta } \\
\text { la carrera }\end{array}$ & NS/NR & \multirow[b]{2}{*}{20} \\
\hline & 3 & 1 & 5 & 11 & \\
\hline \multirow{2}{*}{$\begin{array}{l}\text { Si tuvo dificultades } \\
\text { económicas esto como } \\
\text { determino su retiro }\end{array}$} & Definitivo & Relativo & No influyó & & \multirow[b]{2}{*}{20} \\
\hline & 6 & 6 & 8 & & \\
\hline \multirow{2}{*}{$\begin{array}{l}\text { La U. le ofreció facilidades } \\
\text { de pago para el } \\
\text { financiamiento de la carrera }\end{array}$} & $\mathrm{Si}$ & No & Otro & & \multirow[b]{2}{*}{20} \\
\hline & 4 & 15 & 1 & & \\
\hline
\end{tabular}

\section{Recomendaciones}

Con base en el análisis de la información recopilada, pueden realizarse varias recomendaciones encaminadas a fortalecer la permanencia de los estudiantes en la facultad de enfermería de la Fundación Universitaria de Ciencias de la Salud y prevenir la deserción estudiantil

- En el proceso de selección de los aspirantes a la facultad de enfermería hay que considerar el tiempo transcurrido entre la culminación del bachillerato y el ingreso a la universidad y es preferible que dicho período sea lo más corto posible para así asegurar que los conocimientos sobre las diferentes áreas académicas y el Proyecto Educativo Institucional no se vean interferido por diferentes causas, pues cada institución encamina a los educandos en un área determinada.

- Se sugiere tener en cuenta que en la universidad se debe contar con diferentes áreas recreativas para brindar esparcimiento a los estudiantes y así lograr mejores resultados académicos.

- Es importante evaluar si los aspirantes poseen el perfil que exige la institución, con el propósito de conseguir profesionales convencidos de lo que 
realizan y el país cuente con profesionales más integrales y capaces de enfrentarse a cualquier situación.

- Es de vital importancia contar con campos o sedes adecuadas para la recreación, que no coarten la libertad, lo cual de manera indirecta pero continua afecta la permanencia y estabilidad del alumno dentro de la institución, o aún de manera más accesible, contar con actividades que promuevan la interacción con otras facultades de las diferentes instituciones que se encuentran ubicadas en Bogotá y que proporcionan los espacios que tantos estudiantes demandan por su necesidad de no perder la libertad y perder su juventud por el sedentarismo. Sobre todo, es fundamental que estas actividades se encuentren dentro de las conti- nuas aspiraciones de los estudiantes y no durante determinado tiempo, el cual es muy corto para lograr que los educandos colmen sus expectativas.

- Es conveniente contar con un profesional que oriente a los educandos en los diferentes conflictos a los que ven enfrentados, tanto por su juventud como por su inexperiencia para afrontar los retos y problemas que suelen presentarse durante la permanencia en la universidad.

\section{Bibliografía}

1. Vincent Tinto. Etiología de un sueño.

2. Colombia al filo de la oportunidad. Educación, Ciencia y Tecnología.

3. Pabón Fernández Nohora. Autoevaluación institucional en la perspectiva de la autorregulaciòn. 